OPEN ACCESS

Edited by:

Xiangqian Guo,

Henan University, China

Reviewed by:

Edmund Ui-Hang Sim,

Universiti Malaysia Sarawak, Malaysia

Roopa Biswas,

Uniformed Services University of the

Health Sciences, United States

*Correspondence:

Yongsheng Zhang

shengyongzh@126.com

Juan Wang

wxjyrmyy@163.com

tORCID:

Yongsheng Zhang orcid.org/0000-0002-1857-258X

${ }^{\mp}$ These authors have contributed equally to this work

Specialty section:

This article was submitted to

Cancer Genetics,

a section of the journal

Frontiers in Oncology

Received: 11 August 2020 Accepted: 30 November 2020

Published: 19 January 2021

Citation:

Yang T, Miao X, Bai Z, Tu J, Shen S,

Niu $H$, Xia W, Wang $J$ and Zhang $Y$

(2021) A Novel mRNA-miRNA

Regulatory Sub-Network Associated

With Prognosis of Metastatic Clear Cell

Renal Cell Carcinoma.

Front. Oncol. 10:593601.

doi: 10.3389/fonc.2020.593601

\section{A Novel mRNA-miRNA Regulatory Sub-Network Associated With Prognosis of Metastatic Clear Cell Renal Cell Carcinoma}

\author{
Tianyu Yang ${ }^{1 \neq}$, Xiaofen Miao ${ }^{2 \ddagger}$, Zhanxiang Bai ${ }^{3 \neq}$, Jian $\mathrm{Tu}^{1}$, Shanshan Shen ${ }^{1}$, Hui Niu ${ }^{1}$, \\ Wei Xia ${ }^{1}$, Juan Wang ${ }^{4 *}$ and Yongsheng Zhang ${ }^{1 *+}$ \\ ${ }^{1}$ Department of Pathology, The Second Affiliated Hospital of Soochow University, Suzhou, China, ${ }^{2}$ Department of \\ Pathology, Suzhou TCM Hospital Affiliated to Nanjing University of Chinese Medicine, Suzhou, China, ${ }^{3}$ Department of \\ Pathology, The People's hospital of Hainan Tibetan Autonomous Prefecture, Qinghai, China, ${ }^{4}$ Department of Pathology, \\ Wuzhong People's Hospital of Suzhou, Suzhou, China
}

Background: Clear cell renal cell carcinoma ( $c c R C C)$ is a urinary disease with high incidence. The high incidence of metastasis is the leading cause of death in patients with ccRCC. This study was aimed to identify the gene signatures during the metastasis of ccRCC.

Methods: Two datasets, including one gene expression profile dataset and one microRNA (miRNA) expression profile dataset, were downloaded from Gene Expression Omnibus (GEO) database. The integrated bioinformatics analysis was performed using the (limma) R package, miRWalk, DAVID, STRING, Kaplan-Meier plotter databases. Quantitative real-time polymerase chain reaction (qPCR) was conducted to validate the expression of differentially expressed genes (DEGs) and DE-miRNAs.

Results: In total, 84 DEGs (68 up-regulated and 16 down-regulated) and 41 DE-miRNAs (24 up-regulated and 17 down-regulated) were screened from GSE22541 and GSE37989 datasets, respectively. Furthermore, 11 hub genes and 3 key miRNAs were identified from the PPI network, including FBLN1, THBS2, SCGB1A1, NKX2-1, COL11A1, DCN, LUM, COL1A1, COL6A3, SFTPC, SFTPB, miR-328, miR-502, and miR-504. The qPCR data showed that most of the selected genes and miRNAs were consistent with that in our integrated analysis. A novel mRNA-miRNA network, SFTPBmiR-328-miR-502-miR-504-NKX2-1 was found in metastatic ccRCC after the combination of data from expression, survival analysis, and experiment validation.

Conclusion: In conclusion, key candidate genes and miRNAs were identified and a novel mRNA-miRNA network was constructed in ccRCC metastasis using integrated bioinformatics analysis and qPCR validation, which might be utilized as diagnostic biomarkers and molecular targets of metastatic ccRCC.

Keywords: clear cell renal cell carcinoma, cancer metastasis, bioinformatics analysis, hub genes, miRNAs 


\section{INTRODUCTION}

Renal cell carcinoma (RCC) is a common cancer worldwide, representing approximately $2-3 \%$ of all malignant tumors in adults. It was reported that approximately 63,990 new cases of RCC were diagnosed and more that 14,400 kidney cancer related deaths were found in the United States in 2017 (1, 2). In China, RCC has a rising incidence with about 68,300 new cases of RCC and 25,600 kidney cancer related deaths in 2014 (3). Clear cell RCC (ccRCC) is the most common subtype of RCC and accounts for $80-90 \%$ of RCCs. The metastatic dissemination is the most important factor for the prognosis of ccRCC (4). The 5-year survival rate of patients with primary metastasis is about $10 \%$, and that of patients with non-metastasis and advanced metastasis is $70-90 \%$ (5). At the time of initial diagnosis, nearly $60 \%$ of patients had a local cancer and $20 \%$ had distant metastases $(4,6)$. Since about $30 \%$ of patients with ccRCC develop metastatic diseases after surgery, better understanding of metastatic ccRCC is greatly needed. Metastatic spread of ccRCC mainly occurs in bone, lung, liver, or brain. Metastasis of ccRCC is the cause of its high incidence and poor prognosis. More than $80 \%$ of patients survived less than 5 years after the diagnosis of distant metastasis $(4,7)$. Therefore, necessary researches are needed to elucidate the precise molecular mechanisms how metastatic ccRCC occurs and progresses, and establish new molecular-based strategies to better combat this invasive metastatic ccRCC.

With the rapid development of genomics and transcriptome, more and more evidence shows that non-coding RNAs play important roles in the occurrence, development, and metastasis of various cancers. MicroRNAs (miRNAs) are well known for negatively regulating the expression of its target genes via transcriptional degradation or repression. Many studies have shown that miRNAs play a key regulatory role in the development of human tumors, and affect the progress of cancer by regulating the expression of genes related to the occurrence and development of the disease (8-10). More and more evidence shows that miRNA-mRNA ceRNA network plays a key role in various cancers including pancreatic cancer, gastric cancer, breast cancer, ovarian cancer, liver cancer, and so on (911). However, little is known about the role of miRNA-mRNA ceRNA network in metastatic ccRCC.

In recent years, bioinformatics has become one of the newest fields of biomedical research with the massive amounts of data from high throughout sequencing technologies. Accumulating studies have well documented that bioinformatics analysis have provided a deeper understanding of the aberrant genetic pathways in the development, progression, and metastasis of various human cancers including lung cancer (12), liver cancer (13), ccRCC (1417), and so on. For example, Chen JY et al. identified 14 potential biomarkers for predicting tumorigenesis and progression of ccRCC using weighted gene co-expression network analysis (14). Zhong MY et al. indicated that tumor necrosis factor- $\alpha$-induced protein 8 (TNFAIP8) was highly expressed in ccRCC patients and was associated with the development of advanced ccRCC and poor prognosis by database analysis (15). Bioinformatics analysis of the profiling data by $\mathrm{Ni} \mathrm{D}$ et al. identified FOXO3a as a key factor in
ccRCC metastasis (16). Liang B et al. performed bioinformatic analysis and found that E2F1 and E2F2 may serve as valuable diagnostic markers for renal cancer (17). However, systematic analysis of mRNAs and miRNAs in ccRCC is still not enough. In this study, we performed bioinformatics analysis and identified 11 hub genes [fibulin 1(FBLN1), thrombospondin 2 (THBS2), secretoglobin family $1 \mathrm{~A}$ member 1 (SCGB1A1), NK2 homeobox 1 (NKX2-1), collagen type XI alpha 1 chain (COL11A1), decorin (DCN), lumican (LUM), collagen type I alpha 1 chain (COL1A1), collagen type VI alpha 3 chain (COL6A3), surfactant protein $\mathrm{C}$ (SFTPC), and surfactant protein B (SFTPB)] and three key miRNAs (miR-328, miR-502, and miR-504), and established a novel mRNA-miRNA network in metastatic ccRCC. Based on our current findings, it may be used to determine the prognosis of metastatic ccRCC. They may also serve roles in the early diagnosis and therapy of metastatic ccRCC.

\section{METHODS}

\section{Raw Data}

The datasets used in our study were downloaded from Gene Expression Omnibus (GEO) (http://www.ncbi.nlm.nih.gov/geo/) of the National Center of Biotechnology Information (NCBI) (18). The included studies were regarding comparing the RNAs in metastatic ccRCC tissues and primary ccRCC tissues. The datasets GSE37989 and GSE22541 were obtained in our study. The dataset GSE37989 included miRNA expression profiles of 9 metastatic ccRCC tissues and 12 primary ccRCC tissues, and the platform used to assess is GPL9081 Agilent-016436 Human miRNA Microarray 1.0 G4472A (miRNA ID version). The dataset GSE22541 included mRNA expression profiles of 24 metastatic ccRCC tissues and 24 primary ccRCC tissues, and the platform for these data is GPL570 [HG-U133_Plus_2] Affymetrix Human Genome U133 Plus 2.0 Array.

\section{Identification of Candidate Differential Expression Genes and Differential Expression miRNAs}

To assess candidate DEGs and DE-miRNAs between metastatic ccRCC and primary ccRCC tissues, R language limma package was performed. The Series Matrix Files of these two datasets were downloaded from GEO database, the data were normalized by the normalizeBetweenArray of limma package, and the analysis of DEGs or DE-miRNAs were performed using the (limma) R package (version 3.6.1). $\left|\log _{2} \mathrm{FC}\right|>2$ and $\mathrm{P}$ value $<0.05$ were set as the cut-off criteria. The volcano plots of DEGs or DE-miRNAs were drawn using (ggplot2) $\mathrm{R}$ package (version 3.6.1). The heatmaps of DEGs and DE-miRNAs were drawn by the (heatmap) R package (version 3.6.1).

\section{Prediction of mRNA-miRNA Interactions}

The interactions between DEGs and DE-miRNAs were predicted using miRWalk 3.0 (http://mirwalk.umm.uni-heidelberg.de/) $(19,20)$, which combined the prediction data of both miRDB (21) and Targetscan (22), and a score of $\geqq 0.95$ was considered as 
the cut-off criterion. Only the target genes included in all of the databases were selected for further analysis.

\section{GO and KEGG Enrichment Analysis}

The significant DEGs were enriched by gene ontology (GO) and Kyoto encyclopedia of genes and genomes (KEGG) using DAVID database (https://david.ncifcrf.gov/) (23). P value $<0.05$ was considered as statistically significant. The GO items contained three criteria: biological process (BP), cellular component (CC), and molecular function (MF). The bubble plots of pathway enrichment were drawn using the (ggplot2) R package.

\section{Protein-Protein Interaction Analysis and Hub Genes Identification}

The PPI networks between DEGs were constructed using Search Tool for the Retrieval of Interacting Genes (STRING) database (http://string-db.org/) with a combined score $\geqq 0.4(24,25)$. The regulatory network between DEGs and DE-miRNAs was visualized by Cytoscape software (version 3.6.0) (26). Network analysis was carried out to identify the key hub mRNAs with high degrees in the setup network $(27,28)$, and the top 11 hub genes were visualized in the Cytoscape software based on the node degree.

\section{Survival Analysis}

The Kaplan-Meier plotter database (http://kmplot.com/) (29) was used to evaluate the prognostic values of DEGs and DE-miRNAs. The DEGs and DE-miRNAs were submitted to the database, and the hazard ratio (HR) with 95\% confidence interval and logrank $\mathrm{p}$-value were automatically calculated and directly displayed on the webpage. Logrank p-value $<0.05$ was considered as statistically significant.

\section{Quantitative Real-Time Polymerase Chain Reaction}

Twenty paired ccRCC tissues and the corresponding adjacent non-tumor tissues were obtained from patients with ccRCC. We obtained the written informed consent and the approval from the ethics committee of our Hospital. Total RNA was isolated with the Trizol reagent (Mesgen, China) and the first strand cDNA was synthesized following manufacturer's protocol. The qPCR reactions were conducted in ABI QuantStudio Dx Real-time PCR Detection Systemusing the SYBR-Green-based method based in MIQE guidelines in our study (30). The human $\beta$-actin and human U6 was used as endogenous controls for mRNA and miRNA expression in analysis, respectively. The primers were listed in Supplementary Table 1.

\section{RESULTS}

\section{Identification of Significant Differential Expression Genes and Differential Expression miRNAs in Metastatic ccRCC}

To search the potential differential expression genes (DEGs) and differential expression miRNAs (DE-miRNAs) in metastatic ccRCC tissues compared with primary ccRCC tissues, we conducted a data mining in Gene Expression Omnibus (GEO) database and two datasets (GSE22541 for mRNAs, GSE37989 for miRNAs) were finally included in our study. Next, the differential expression analysis was performed to find the significant DEGs and DE-miRNAs in each GEO dataset by using the limma package with the criterion of $|\log 2 \mathrm{FC}|>2$ and $\mathrm{P}$ value $<0.05$. Finally, 84 significant DEGs were found in GSE22541 dataset with 68 up-regulated and 16 down-regulated genes, and 41 significant DE-miRNAs were obtained in GSE37989 dataset with 24 up-regulated and 17 down-regulated miRNAs (Supplementary Tables 2-4). The volcano plots and heatmaps of significant DEGs and DE-miRNAs were shown in Figures 1A-D.

\section{Functional Enrichment Analysis for the Significant Differential Expression Genes}

To predict the potential biological functions and related pathways of these DEGs, GO and KEGG assay was conducted. As shown in Figure 2A, the DEGs were significant enriched in cancer associated pathways including surfactant metabolism, signaling by receptor tyrosine kinases, signaling by PDGF, non-integrin membrane-ECM interactions, MET activates PTK2 signaling, and so on. Furthermore, the enriched GO functions included visual perception, respiratory gaseous exchange, negative regulation of cell adhesion, multicellular organismal process, extracellular matrix organization in the $\mathrm{BP}$ category (Figure 2B); supramolecular fiber, multivesicular body lumen, fibrillary collagen trimer, extracellular region part, extracellular matrix component in the CC category (Figure 2C); peptidase regulatory activity, heparin binding, glycosaminoglycan binding extracellular matrix structural constituent, extracellular matrix binding in the MF category (Figure 2D).

\section{Protein-Protein Interaction Analysis of miRNA-mRNA Network}

The PPI networks of the significant DEGs were constructed using STRING database, and the interactions of DEGs and DEmiRNAs were analyzed by miRWalk 3.0 with the criterion of a score of $\geqq 0.95$. The network of DEGs and DE-miRNA were rebuilt and visualized by Cytoscape software. As shown in Figure 3, there were 101 nodes in miRNA-mRNA network, which consisted of 84 mRNAs, 27 miRNAs, and formed 283 connections. In addition, the top 11 hub genes were identified from these significant DEGs based on node degree (Supplementary Table 5). All the hub genes were upregulated including FBLN1, THBS2, SCGB1A1, NKX2-1, COL11A1, DCN, LUM, COL1A1, COL6A3, SFTPC, and SFTPB. The regulatory network of hub genes/miRNAs was shown in Figure 3. There were 27 nodes in miRNA-mRNA network, which consisted of 11 mRNAs, 16 miRNAs, and formed 53 connections. Among the hub genes/miRNAs network, the down-regulated miRNAs were hsa-miR-452*, hsa-miR-502, hsa-miR-377, hsa-miR-423, hsa-miR-136, 


\section{A MRNA}

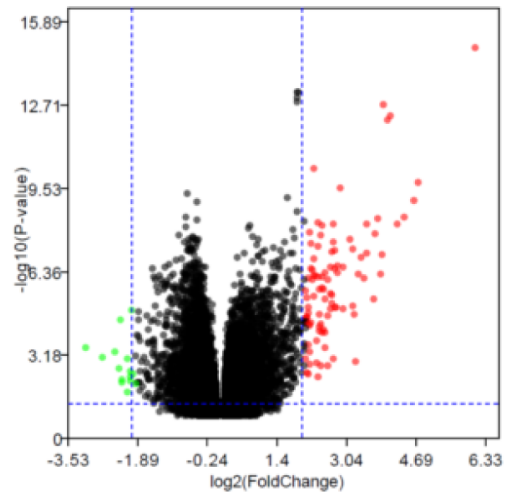

C mRNA

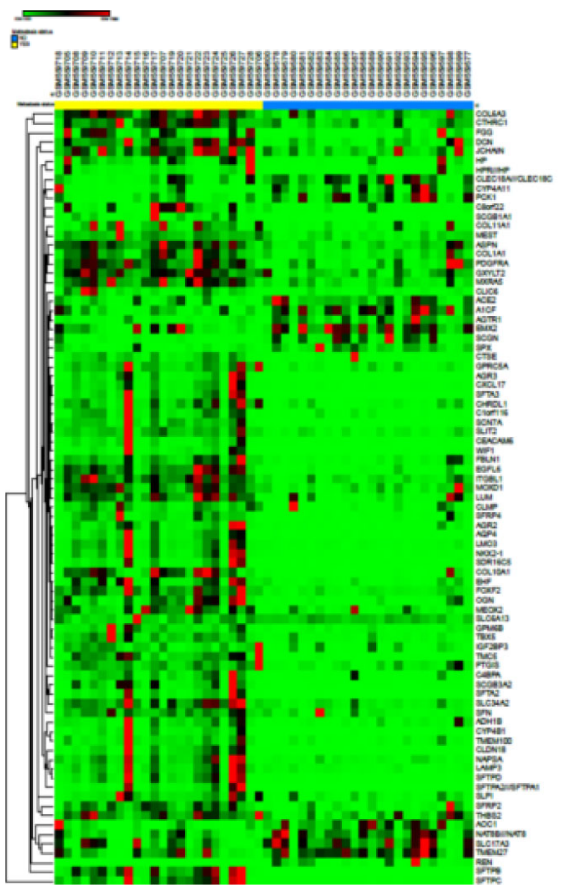

B miRNA

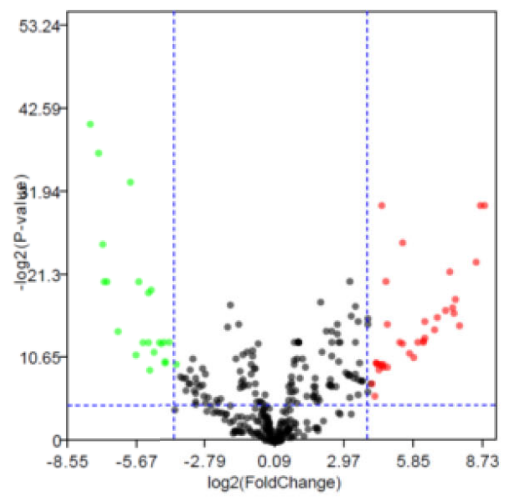

D miRNA

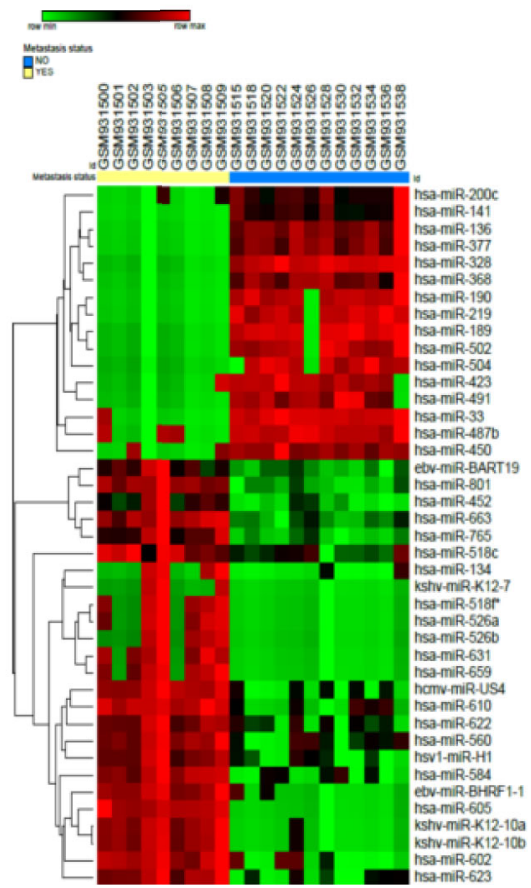

FIGURE 1 | Differentially expressed genes and miRNAs in metastatic ccRCC and primary ccRCC tissues. (A) Volcano plot of differentially expressed mRNAs from GSE22541 dataset; (B) Volcano plot of differentially expressed miRNAs from GSE37989 dataset; (C) Heatmap of differentially expressed mRNAs from GSE22541 dataset; (D) Heatmap of differentially expressed miRNAs from GSE37989 dataset.

hsa-miR-328, and hsa-miR-504, whose target genes were SFTPB, SFTPC, NKX2-1, FBLN1, COL11A1, DCN, and COL1A1, and the up-regulated miRNAs were hsa-miR-518c, hsa-miR-584, hsa-miR-134, hsa-miR-623, hsa-miR-765, hsamiR-663a, hsa-miR-526b, hsa-miR-622, and hsa-miR-605, whose target genes were COL1A1, DCN, SCGB1A1, NKX2-1, SFTPB, and FBLN1 (Figure 4). Based on the classical inverse relationship between miRNAs and target genes, the upregulated 11 hub genes and their corresponding downregulated miRNAs were used for further analysis considering that the regulation of genes and miRNAs.

\section{Validation of Hub Genes and Key miRNAs in Metastatic ccRCC by Prognostic Evaluation}

In order to further identify key genes in metastatic ccRCC, we detected the prognostic values of the hub genes using KaplanMeier plotter database. As depicted in Figures $\mathbf{5 A - K}$, the increased expression of ten hub genes (FBLN1, THBS2, SCGB1A1, NKX2-1, COL11A1, DCN, LUM, COL1A1, COL6A3, and SFTPC) indicated poor prognosis in 530 patients with ccRCC (all $\mathrm{P}<0.01$ ). Meanwhile, the KaplanMeier plotter database was also used to detect the prognostic 

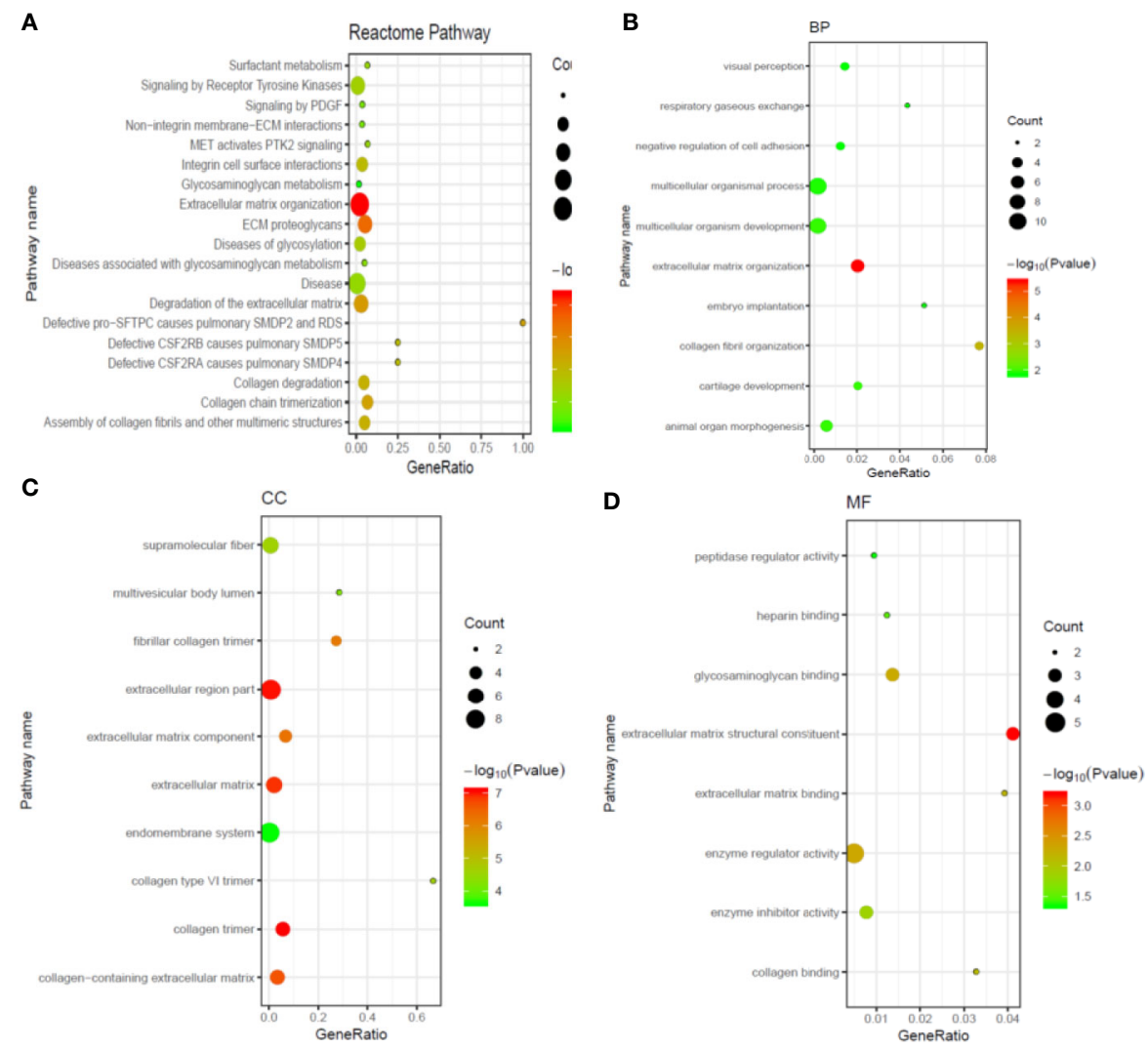

FIGURE 2 | Gene ontology (GO) and Kyoto Encyclopedia of Genes and Genome (KEGG) enrichment analysis for functions of differentially expressed genes in metastatic ccRCC. (A) The top 20 most significant KEGG pathway terms; (B) The top 10 most significant changes in the GO biological process; (C) The top 10 most significant changes in the GO cellular component; (D) The top 10 most significant changes in the GO molecular function.

values of these miRNAs, which were down-regulated in metastatic ccRCC tissues, and could potentially regulate the expression of the above hub genes. As shown in Figures 6AG, the high expression of miR-328, miR-502, and miR-504 exhibited favorable prognostic values in ccRCC with HR values of $0.7(0.52-0.96, \mathrm{P}=0.026), 0.67(0.49-0.92, \mathrm{P}=0.011)$, and 0.55 $(0.4-0.74, \mathrm{P}=0.000058)$, respectively. Combined the above results of expression and survival analysis for key miRNAs, we re-defined the three key miRNAs (miR-328, miR-502, and miR504) as the key miRNAs.

\section{qPCR Validation of Hub Genes and Key miRNAs}

The hub genes and key miRNAs were also validated by qPCR using 20 paired ccRCC tissues and adjacent normal kidney tissues. As shown in Figures 7A-N, the expression of SFTPB, THBS2, SCGB1A1, NKX2-1, COL11A1, DCN, and COL1A1 increased significantly in ccRCC tissues compared with adjacent normal kidney tissues $(P<0.05, P<0.01)$ and the expression of SFTPC, FBLN1,LUM, and COL6A3 increased in ccRCC tissues, while the levels of miR-328, miR-502, and miR-504 greatly decreased in ccRCC tissues $(P<0.01)$. Generally, the qPCR results were consistent with that in our integrated analysis.

\section{Construction of Key mRNA-miRNA Sub-Network in Metastatic ccRCC}

A key mRNA-miRNA competitive endogenous RNA regulatory network in metastatic ccRCC was constructed by a series of data mining and analysis. The network consisted of four mRNAmiRNA pairs (SFTPB-miR-328, SFTPB-miR-502, SFTPB-miR504, and NKX2-1-miR-504), which was depicted in Figure 8. Taken together, we constructed a novel mRNA-miRNA subnetwork, SFTPB-miR-328-miR-502-miR-504-NKX2-1, which was obviously associated with the metastatic capacity and prognosis of ccRCC. The sub-network may be developed as promising diagnostic biomarkers or therapeutic targets for metastatic ccRCC in the future.

\section{DISCUSSION}

In recent years, accumulating preclinical and clinical studies have revealed the potential mechanism of metastatic ccRCC, but the incidence and mortality of metastatic ccRCC remain high (16, 31,32 ). This is mainly because most studies focus on a single genetic change, or the data based on a single cohort study. In this study, we analyzed two datasets from GEO database and 


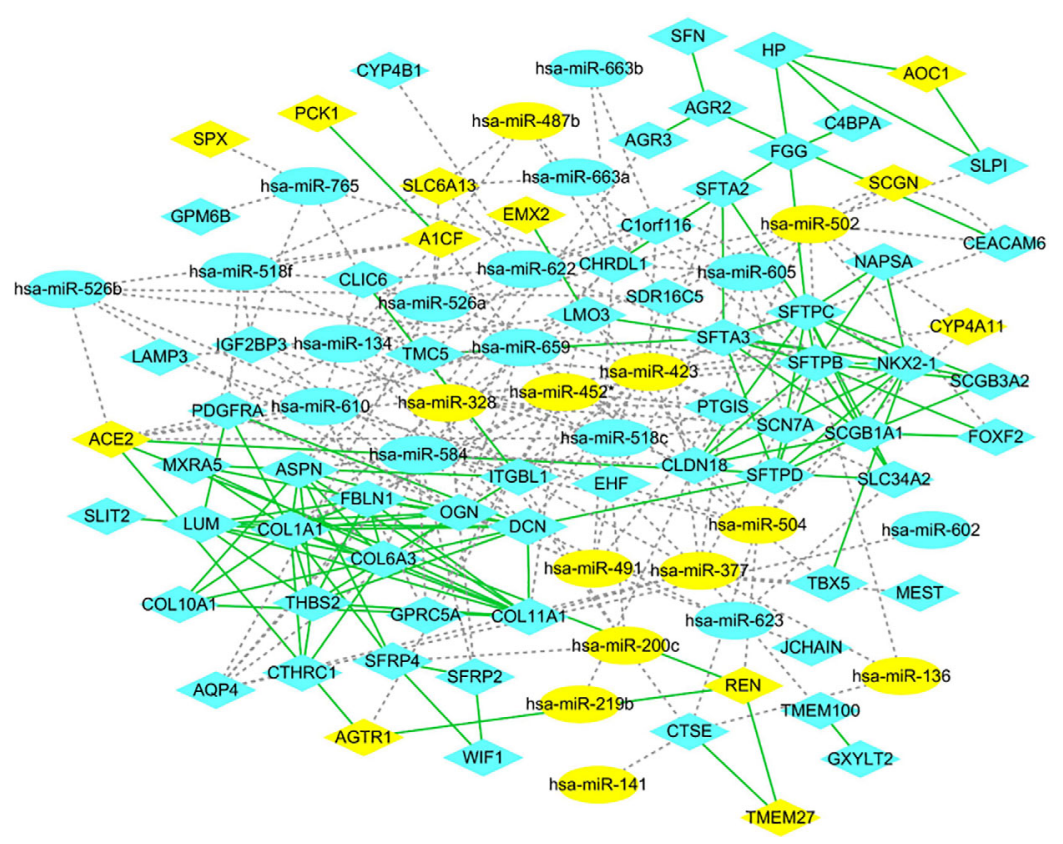

FIGURE 3 | Protein-protein interaction (PPI) network. mRNAs and miRNAs are indicated as ellipsoid and diamond, respectively. The gray dotted line represents the interaction of the miRNA-mRNAs. The green line represents the interaction of the proteins. The yellow indicates high expression, and blue indicates low expression.

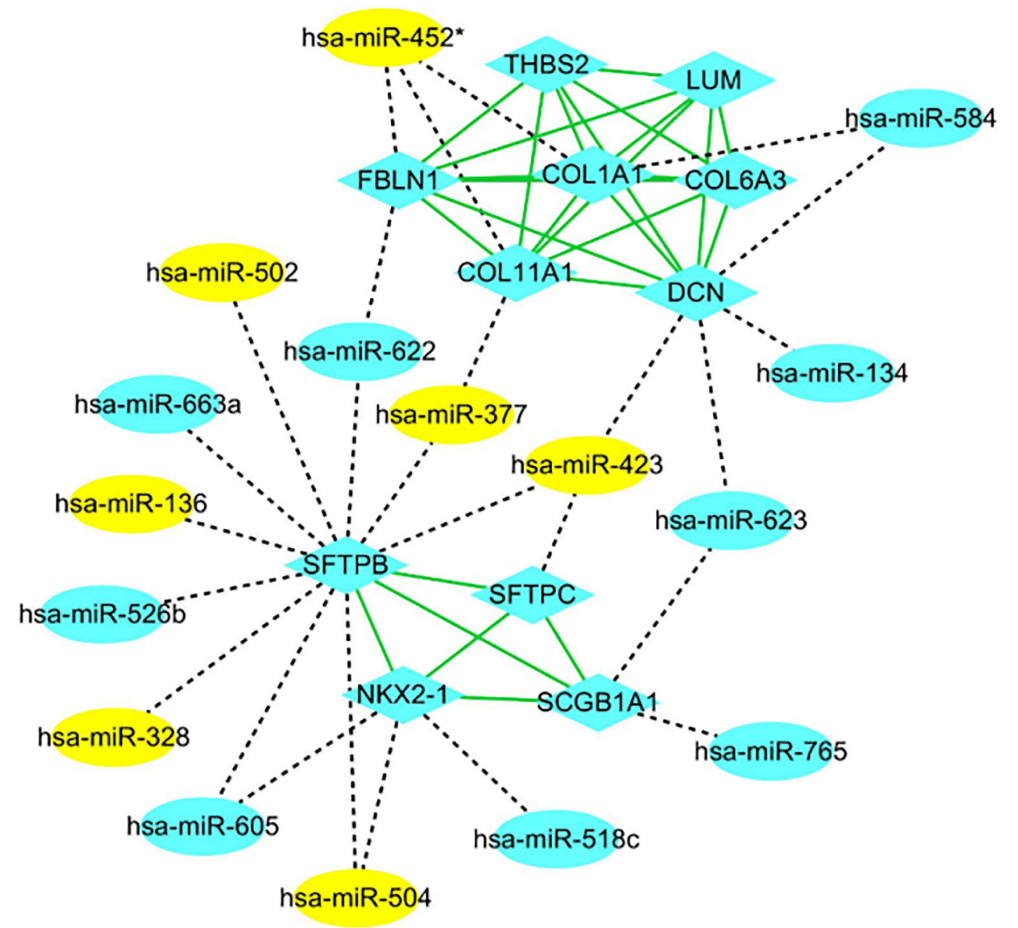

FIGURE 4 | PPI network of hub genes and key miRNAs. mRNAs and miRNAs are indicated as ellipsoid and diamond, respectively. The gray dotted line represents the interaction of the miRNA-mRNAs. The green line represents the interaction of the proteins. The yellow indicates high expression, and blue indicates low expression. * indicates hsa-miR-452-3p. 

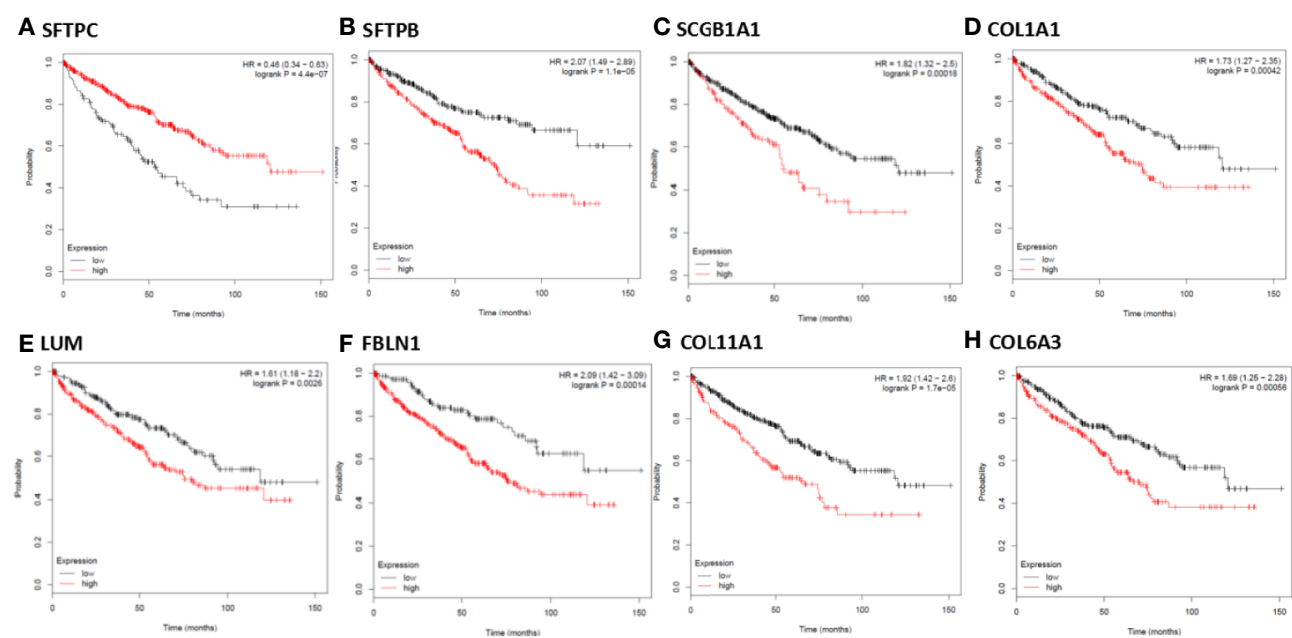

G COL11A1

\section{H COL6A3}
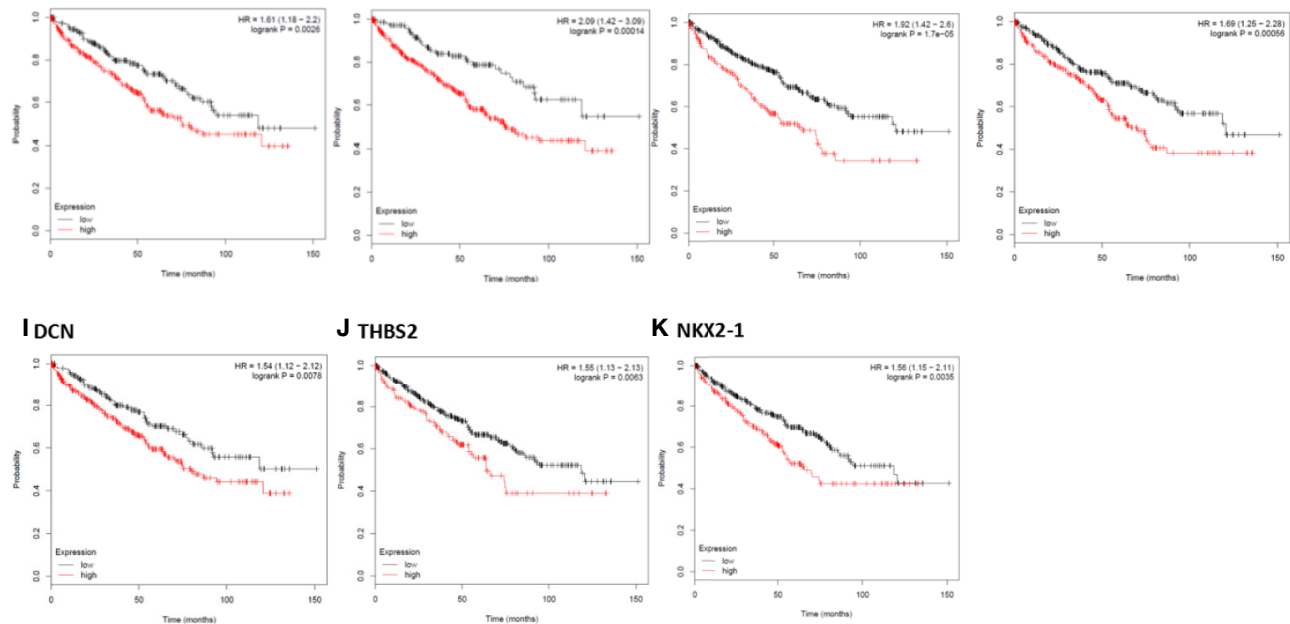

FIGURE 5 | Kaplan-Meier survival analysis for the correlation of 11 hub genes with overall survival of the patients with ccRCC. The vertical coordinate represents the survival probability of cCRCC patients. The red curve represents CcRCC patients with up-regulation of genes, while the black curve represents ccRCC patients with down-regulation of genes.

identified 84 DEGs (68 up-regulated and 16 down-regulated), and 41 DE-miRNAs (24 up-regulated and 17 down-regulated). The following GO item and KEGG were used to enrich and analyze differentially expressed genes, and PPI network was constructed. Finally, top 11 hub genes were identified, and a novel mRNA-miRNA regulatory network was constructed, and every RNA in this network exhibited an obvious prognostic value in ccRCC.

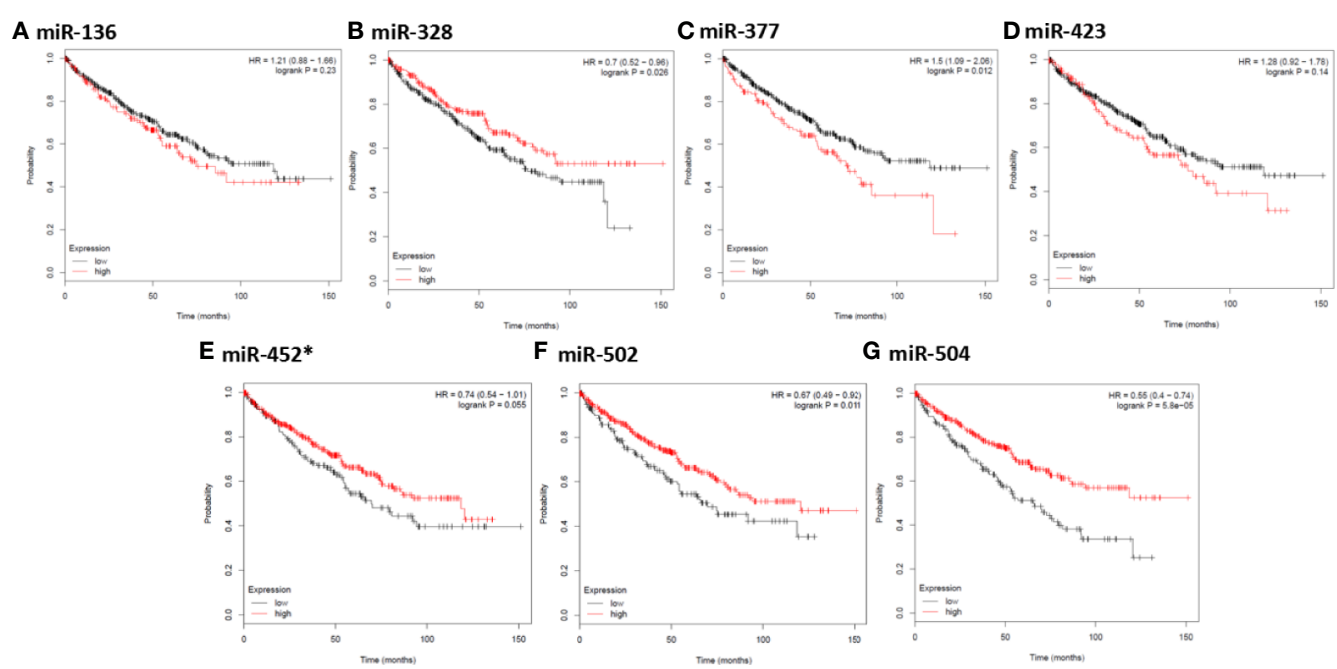

FIGURE 6 | Kaplan-Meier survival analysis for the correlation of seven down-regulated key miRNAs with overall survival of the patients with ccRCC. The vertical coordinate represents the survival probability of ccRCC patients. The red curve represents ccRCC patients with up-regulation of miRNAs, while the black curve represents ccRCC patients with down-regulation of miRNAs. * indicates hsa-miR-452-3p. 


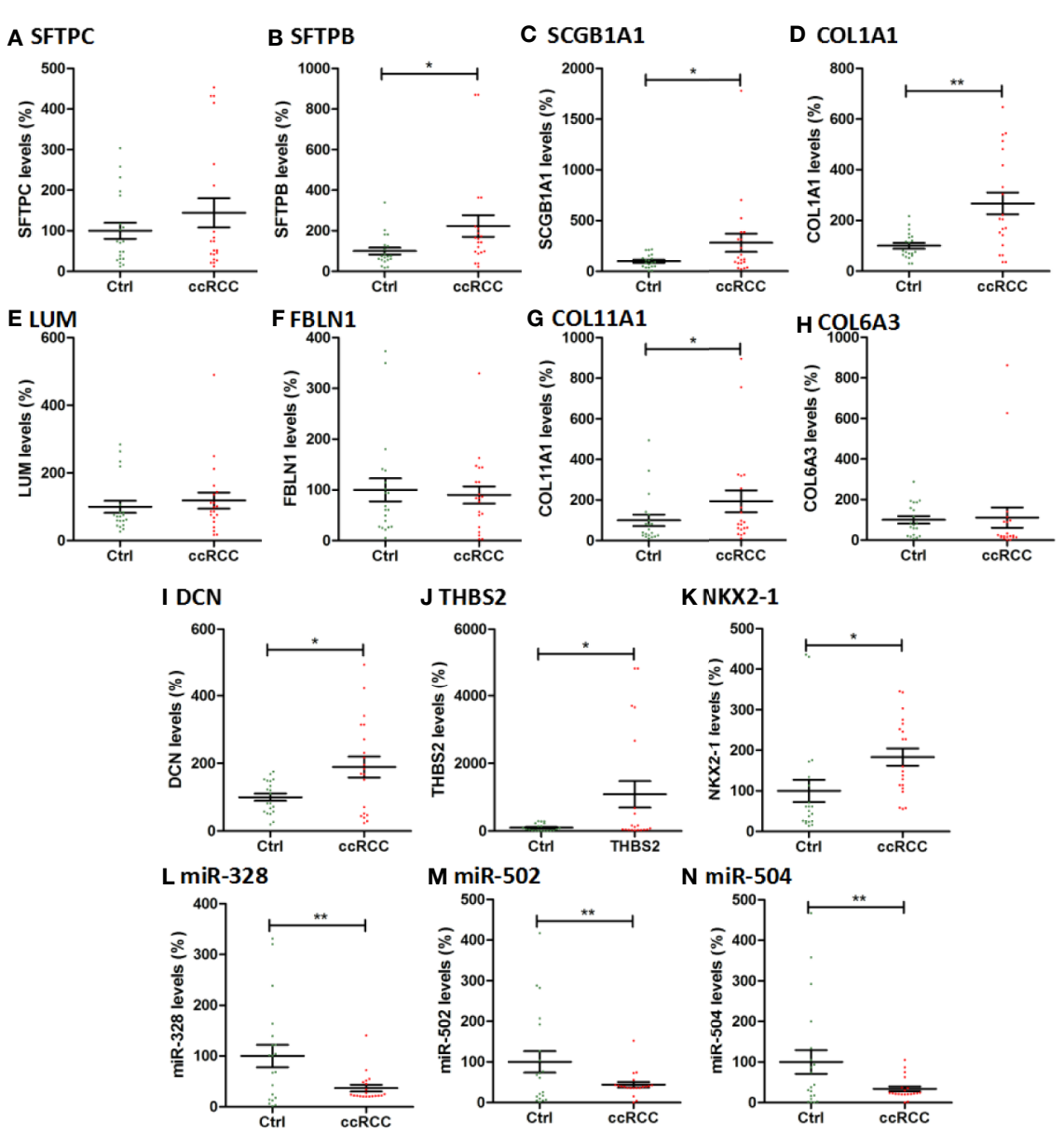

FIGURE 7 | The qPCR results of 11 hub genes and 3 key miRNAs in 20 ccRCC tissues and paired adjacent normal kidney tissues. The vertical coordinate represents the expression levels of genes and miRNAs. Ctrl: Paracancer tissues. ${ }^{*}$ indicates $\mathrm{P}<0.05$; ${ }^{\star *}$ indicates $\mathrm{P}<0.01$.

After data mining, integrated bioinformatics analysis, and qPCR validation, 11 hub genes were found including FBLN1, THBS2, SCGB1A1, NKX2-1, COL11A1, DCN, LUM, COL1A1, COL6A3, SFTPC, and SFTPB. Among these genes, COL11A1, COL1A1, COL6A3 belong to the collagen family, and are primarily involved in organizing functions through binding to cellular receptors and other components of the extracellular matrix (ECM) (33). COL1A1 is the pro-alphal chains of type I collagen whose triple helix comprises two alpha1 chains and one alpha2 chain. COL1A1 is an ECM protein, whose overexpression was linked to breast cancer (34), gastric cancer (34), and colorectal cancers (35). COL1A1 was found to be overexpressed in non-small cell lung cancer (NSCLC) tissues, and COL1A1 correlated with hypoxia markers in NSCLC (36). Moreover, miR-129-5p suppresses gastric cancer cell proliferation, migration, and invasion, by selectively inhibiting COL1A1 (37). COL11A1 is a minor fibrillar collagen. COL11A1 was overexpressed in bowel metastases among patients with ovarian cancer (38), and promoted ovarian cancer progression and is associated with chemo-resistance to cisplatin and paclitaxel in ovarian cancer cells (39). COL6A3 gene silencing inhibits gastric cancer cell proliferation, migration, and invasion while promoting apoptosis through the PI3K-Akt signaling pathway (40). However, the roles of COL11A1, COL1A1, and COL6A3 in the tumorigenesis and metastasis of ccRCC remains to be clarified. Our integrated bioinformatics analysis demonstrated a significant up-regulation of COL11A1, COL1A1, and COL6A3 in metastatic ccRCC tissues, suggesting a proto-oncogene effect of COL11A1, COL1A1, and COL6A3 in ccRCC metastasis. FBLN1 was observed to significantly down-regulated in RCC cell lines and patient tissues through promoter hypermethylation, and FBLN1 over expression led to decreased cell growth, enhanced tumor cell apoptosis, decreased cell motility, and angiogenesis of RCC cells in vitro and in vivo (41). DCN expression was decreased in RCC tissues compared to adjacent noncancerous tissues and was highly correlated to tumor size (42). In addition, DCN overexpression could inhibit RCC cell proliferation and metastasis by the upregulating of p21 and E-cadherin (42). Additionally, the roles of THBS2, SCGB1A1, NKX2-1, LUM, SFTPC, and SFTPB in the development and metastasis of ccRCC are unclear. Thus, more researches are needed to further demonstrate the roles and molecular mechanisms of the above hub genes in the metastasis of ccRCC. 


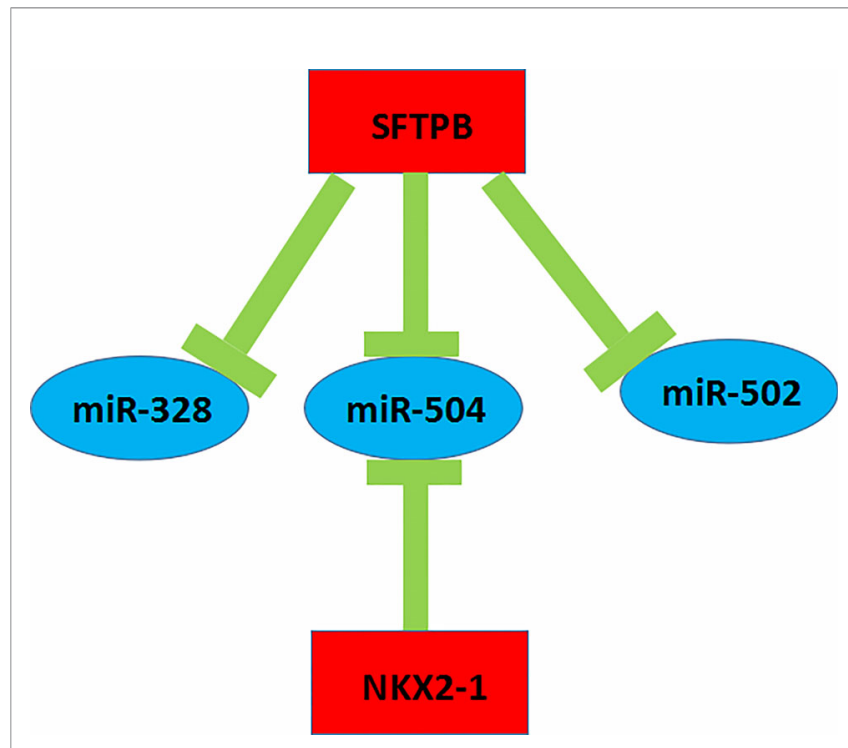

FIGURE 8 | The novel mRNA-miRNA regulatory network associated with prognosis of the patients with cCRCC.

A large number of studies have shown that miRNAs play key regulatory roles in the development, metastasis, and progression of human tumors including ccRCC $(8-11,31)$. MiRNAs not only play the role of proto-oncogenes in tumor cells by promoting the occurrence and development of tumors, but also play a role of anti-cancer via inhibiting the oncogenesis and metastasis of cancers. Our integrated bioinformatics analysis identified three down-regulated DE-miRNAs (miR328, miR-502, and miR-504) in metastatic ccRCC tissues. Moreover, the expression and prognosis of these three miRNAs were further confirmed between ccRCC tissues and normal kidney tissues, suggesting the tumor suppressor role of these miRNAs. Consistent with our current study, recent studies demonstrated the tumor suppressor role of miR-328 and miR-502. miR-328 was found to inhibit the growth of renal cancer cells by regulating cellular adhesion and migration in RCC (43). A recent study showed that knock down of histone methyltransferase SET8, a target gene of miR-502, led to the inhibition of cell proliferation, colony formation, cellular migration, and invasion in RCC cells, implying the anticancer activity of miR-502 (44). In addition, miR-504 has been reported to play a tumor suppressor role in the oncogenesis and metastasis of malignant cancers. Liu Q et al. reported that miR-504 suppressed mesenchymal phenotype and invasion of glioblastoma by targeting Frizzled-7 and inhibiting Wnt/ $\beta$-catenin signaling (45). Ye MF et al. showed that miR-504 was notably down-regulated in non-small cell lung cancer (NSCLC) tissues, and the up-regulation of miR-504 significantly inhibited cell proliferation, cell invasion, and EMT process of NSCLC by directly targeting LOXL2 gene (46). Although multiple studies have suggested the role of miR-504 in cancer development and metastasis, the exact role and mechanisms of miR-504 in ccRCC remains largely unknown yet, which requires further investigation.
Establishment of miRNA-mRNA regulatory networks may provide key evidences to investigate the molecular mechanisms of malignant cancers. For example, Wang WL et al. uncovered key prognostic biomarkers for pancreatic cancer through identification of a novel mRNA-miRNA-lncRNA sub-network (27). Ding BS et al. constructed a potential circRNA-miRNAmRNA ceRNA network for colorectal cancer (47); Zhang JJ et al. also identified a mRNA-miRNA-lncRNA ceRNA network associated with diagnosis and prognosis of hepatocellular carcinoma (48). The above studies established miRNA-mRNA sub-network utilizing high throughout RNA data in cancer patients. In this study, in order to reveal the possible regulatory network in metastatic ccRCC, we constructed a DEmiRNAs-DEGs regulatory network, in which, SFTPB-miR-328, SFTPB-miR-502, SFTPB-miR-504, and NKX2-1-miR-504 axes may play an important role in the occurrence and metastasis of ccRCC. Intriguingly, most of these key genes have been well investigated in cancers. For example, SFTPB is major component of pulmonary surfactant and is secreted by both alveolar type II and club lung epithelial cells. SFTPB is over expressed in nonsmall cell lung cancer, especially in lung adeno-carcinoma (49). Moreover, pro-SFTPB in plasma, the precursor of SFTPB, was an independent predictor of lung cancer (50). Clinical studies showed that low NKX2-1 level correlated with unfavorable prognoses in lung adenocarcinoma patients (51). The above studies partially support the accuracy of our bioinformatic analysis. Therefore, more studies are needed to investigate the role of SFTPB, NKX2-1 and their axes in the oncogenesis and metastasis of ccRCC.

In conclusion, our study identified 11 hub genes (FBLN1, THBS2, SCGB1A1, NKX2-1, COL11A1, DCN, LUM, COL1A1, COL6A3, SFTPC, SFTPB) and 3 key miRNAs (miR-328, miR502, miR-504). Furthermore, we constructed a DE-miRNAsDEGs regulatory network, which may be helpful for us to clarify the molecular mechanisms of metastasis of ccRCC. Our study provides several key clues to the molecular mechanistic study of metastatic ccRCC.

\section{DATA AVAILABILITY STATEMENT}

The datasets presented in this study can be found in online repositories. The names of the repository/repositories and accession number(s) can be found in the article/Supplementary Material.

\section{ETHICS STATEMENT}

The studies involving human participants were reviewed and approved by the ethics committee of The Second Affiliated Hospital of Soochow University. The patients/participants provided their written informed consent to participate in this study. Written informed consent was obtained from the individual(s) for the publication of any potentially identifiable images or data included in this article. 


\section{AUTHOR CONTRIBUTIONS}

TY and YZ conceived, designed, and wrote the manuscript. TY, XM, $\mathrm{ZB}$, JT, and JW performed the experiments and analyzed the data. SS and HN helped with the manuscript and data review. All authors contributed to the article and approved the submitted version.

\section{FUNDING}

This study was supported by funds from Suzhou Science and Technology Development Program (SYS201717), the Second

\section{REFERENCES}

1. Siegel RL, Miller KD, Jemal A. Cancer statistics, 2018. CA Cancer J Clin (2018) 68:7-30. doi: 10.3322/caac. 21442

2. Scelo G, Larose TL. Epidemiology and Risk Factors for Kidney Cancer. J Clin Oncol (2018) 36(36):JCO2018791905. doi: 10.1200/JCO.2018.79.1905

3. Liu SZ, Guo LW, Cao XQ, Chen Q, Zhang SK, Zhang M, et al. [Estimation on the incidence and mortality of kidney cancer in China, in 2014]. Zhonghua Liu Xing Bing Xue Za Zhi (2018) 39:1346-50. doi: 10.3760/cma.j.issn.02546450.2018.10.011

4. Nguyen DX, Bos PD, Massague J. Metastasis: from dissemination to organspecific colonization. Nat Rev Cancer (2009) 9:274-84. doi: 10.1038/nrc2622

5. Ljungberg B. Prognostic factors in renal cell carcinoma. Urol A (2004) 43 (Suppl 3):S119-20. doi: 10.1007/s00120-004-0594-6

6. Wotschofsky Z, Liep J, Meyer HA, Jung M, Wagner I, Disch AC, et al. Identification of metastamirs as metastasis-associated microRNAs in clear cell renal cell carcinomas. Int J Biol Sci (2012) 8:1363-74. doi: 10.7150/ijbs.5106

7. Cheng SK, Chuah KL. Metastatic Renal Cell Carcinoma to the Pancreas: A Review. Arch Pathol Lab Med (2016) 140:598-602. doi: 10.5858/arpa.20150135-RS

8. Motti ML, AS D, Meccariello R. MicroRNAs, Cancer and Diet: Facts and New Exciting Perspectives. Curr Mol Pharmacol (2018) 11:90-6. doi: 10.2174/ 1874467210666171013123733

9. Vannini I, Fanini F, Fabbri M. Emerging roles of microRNAs in cancer. Curr Opin Genet Dev (2018) 48:128-33. doi: 10.1016/j.gde.2018.01.001

10. Kai K, Dittmar RL, Sen S. Secretory microRNAs as biomarkers of cancer. Semin Cell Dev Biol (2018) 78:22-36. doi: 10.1016/j.semcdb.2017.12.011

11. Liu W, Lv C, Zhang B, Zhou Q, Cao Z. MicroRNA-27b functions as a new inhibitor of ovarian cancer-mediated vasculogenic mimicry through suppression of VE-cadherin expression. RNA (2017) 23:1019-27. doi: 10.1261/rna.059592.116

12. Wu D, Wang X. Application of clinical bioinformatics in lung cancer-specific biomarkers. Cancer Metastasis Rev (2015) 34:209-16. doi: 10.1007/s10555015-9564-2

13. Zheng C, Zheng L, Yoo JK, Guo H, Zhang Y, Guo X, et al. Landscape of Infiltrating T Cells in Liver Cancer Revealed by Single-Cell Sequencing. Cell (2017) 169:1342-56.e16. doi: 10.1016/j.cell.2017.05.035

14. Chen JY, Sun Y, Qiao N, Ge YY, Li JH, Lin Y, et al. Co-expression Network Analysis Identifies Fourteen Hub Genes Associated with Prognosis in Clear Cell Renal Cell Carcinoma. Curr Med Sci (2020) 40:773-85. doi: 10.1007/ s11596-020-2245-6

15. Zhong M, Zhu M, Liu Y, Lin Y, Wang L, Ye Y, et al. TNFAIP8 promotes the migration of clear cell renal cell carcinoma by regulating the EMT. J Cancer (2020) 11:3061-71. doi: 10.7150/jca.40191

16. Ni D, Ma X, Li HZ, Gao Y, Li XT, Zhang Y, et al. Downregulation of FOXO3a promotes tumor metastasis and is associated with metastasis-free survival of patients with clear cell renal cell carcinoma. Clin Cancer Res (2014) 20:177990. doi: 10.1158/1078-0432.CCR-13-1687

17. Liang B, Zhao J, Wang X. Clinical performance of E2Fs 1-3 in kidney clear cell renal cancer, evidence from bioinformatics analysis. Genes Cancer (2017) 8:600-7. doi: 10.18632/genesandcancer.143
Affiliated Hospital of Soochow University Advance Research Program of the National Natural Science Foundation of China grants (SDFEYGJ1705), and the Advance Research Program of the Second Affiliated Hospital of Soochow University (SDFEYBS1806, SDFEYQN1801).

\section{SUPPLEMENTARY MATERIAL}

The Supplementary Material for this article can be found online at: https://www.frontiersin.org/articles/10.3389/fonc.2020. 593601/full\#supplementary-material

18. Barrett T, Wilhite SE, Ledoux P, Evangelista C, Kim IF, Tomashevsky M, et al. NCBI GEO: archive for functional genomics data sets-update. Nucleic Acids Res (2013) 41:D991-95. doi: 10.1093/nar/gks1193

19. Dweep H, Gretz N, Sticht C. miRWalk database for miRNA-target interactions. Methods Mol Biol (2014) 1182:289-305. doi: 10.1007/978-14939-1062-5_25

20. Sticht C, De La Torre C, Parveen A, Gretz N. miRWalk: An online resource for prediction of microRNA binding sites. PloS One (2018) 13:e0206239. 00000003-3596-6094 AO. doi: 10.1371/journal.pone.0206239

21. Chen Y, Wang X. miRDB: an online database for prediction of functional microRNA targets. Nucleic Acids Res (2020) 48:D127-127D131. doi: 10.1093/ nar/gkz757

22. Franceschini A, Szklarczyk D, Frankild S, Kuhn M, Simonovic M, Roth A, et al. STRING v9.1: protein-protein interaction networks, with increased coverage and integration. Nucleic Acids Res (2013) 41:D808-15. doi: 10.1093/ nar/gks1094

23. Dennis GJr, Sherman BT, Hosack DA, Yang J, Gao W, Lane HC, et al. DAVID: Database for Annotation, Visualization, and Integrated Discovery. Genome Biol (2003) 4:P3. doi: 10.1186/gb-2003-4-9-r60

24. Szklarczyk D, Morris JH, Cook H, Kuhn M, Wyder S, Simonovic M, et al. The STRING database in 2017: quality-controlled protein-protein association networks, made broadly accessible. Nucleic Acids Res (2017) 45:D362362D368. doi: 10.1093/nar/gkw937

25. Quillet A, Saad C, Ferry G, Anouar Y, Vergne N, Lecroq T, et al. Improving Bioinformatics Prediction of microRNA Targets by Ranks Aggregation. Front Genet (2019) 10:1330. doi: 10.3389/fgene.2019.01330

26. Kohl M, Wiese S, Warscheid B. Cytoscape: software for visualization and analysis of biological networks. Methods Mol Biol (2011) 696:291-303. doi: 10.1007/978-1-60761-987-1_18

27. Wang W, Lou W, Ding B, Yang B, Lu H, Kong Q, et al. A novel mRNAmiRNA-lncRNA competing endogenous RNA triple sub-network associated with prognosis of pancreatic cancer. Aging (Albany NY) (2019) 11:2610-27. doi: 10.18632/aging.101933

28. Baruah MM, Sharma N. AUID- Oho. In silico identification of key genes and signaling pathways targeted by a panel of signature microRNAs in prostate cancer. Med Oncol (2019) 36:43. doi: 10.1007/s12032-019-1268-y

29. Gyorffy B, Lanczky A, Eklund AC, Denkert C, Budczies J, Li Q, et al. An online survival analysis tool to rapidly assess the effect of 22,277 genes on breast cancer prognosis using microarray data of 1,809 patients. Breast Cancer Res Treat (2010) 123:725-31. doi: 10.1007/s10549-009-0674-9

30. Bustin SA, Benes V, Garson JA, Hellemans J, Huggett J, Kubista M, et al. The MIQE guidelines: minimum information for publication of quantitative realtime PCR experiments. Clin Chem (2009) 55:611-22. doi: 10.1373/ clinchem.2008.112797

31. Wu X, Weng L, Li X, Guo C, Pal SK, Jin JM, et al. Identification of a 4microRNA signature for clear cell renal cell carcinoma metastasis and prognosis. PloS One (2012) 7:e35661. doi: 10.1371/journal.pone.0035661

32. White NM, Khella HW, Grigull J, Adzovic S, Youssef YM, Honey RJ, et al. miRNA profiling in metastatic renal cell carcinoma reveals a tumoursuppressor effect for miR-215. Br J Cancer (2011) 105:1741-49. doi: 10.1038/bjc. 2011.401 
33. Bella J, Hulmes DJ. Fibrillar Collagens. Subcell Biochem (2017) 82:457-90. doi: 10.1007/978-3-319-49674-0_14

34. Jansen JT, Veldkamp WJ, Thijssen MA, van Woudenberg S, Zoetelief J. Method for determination of the mean fraction of glandular tissue in individual female breasts using mammography. Phys Med Biol (2005) 50:5953-67. doi: 10.1088/0031-9155/50/24/013

35. Zhang Z, Fang C, Wang Y, Zhang J, Yu J, Zhang Y, et al. COL1A1: A potential therapeutic target for colorectal cancer expressing wild-type or mutant KRAS. Int J Oncol (2018) 53:1869-80. doi: 10.3892/ijo.2018.4536

36. Oleksiewicz U, Oho AUID-, Liloglou T, Tasopoulou KM, Daskoulidou N, Gosney JR, et al. COL1A1, PRPF40A, and UCP2 correlate with hypoxia markers in non-small cell lung cancer. J Cancer Res Clin Oncol (2017) 143:1133-41. doi: 10.1007/s00432-017-2381-y

37. Wang Q, Yu J. MiR-129-5p suppresses gastric cancer cell invasion and proliferation by inhibiting COL1A1. Biochem Cell Biol (2018) 96:19-25. doi: $10.1139 / \mathrm{bcb}-2016-0254$

38. Mariani A, Wang C, Oberg AL, Riska SM, Torres M, Kumka J, et al. Genes associated with bowel metastases in ovarian cancer. Gynecol Oncol (2019) 154:495-504. doi: 10.1016/j.ygyno.2019.06.010

39. Wu YH, Huang YF, Chang TH, Chou CY. Activation of TWIST1 by COL11A1 promotes chemoresistance and inhibits apoptosis in ovarian cancer cells by modulating NF-kappaB-mediated IKKbeta expression. Int $\mathrm{J}$ Cancer (2017) 141:2305-17. 0000-0002-3672-6917 AO. doi: 10.1002/ijc.30932

40. Ao R, Guan L, Wang Y, Wang JN. Silencing of COL1A2, COL6A3, and THBS2 inhibits gastric cancer cell proliferation, migration, and invasion while promoting apoptosis through the PI3k-Akt signaling pathway. J Cell Biochem (2018) 119:4420-34. 0000-0001-7672-1793 AO. doi: 10.1002/jcb.26524

41. Xiao W, Wang J, Li H, Guan W, Xia D, Yu G, et al. Fibulin-1 is downregulated through promoter hypermethylation and suppresses renal cell carcinoma progression. J Urol (2013) 190:291-301. doi: 10.1016/ j.juro.2013.01.098

42. Xu Y, Xia Q, Rao Q, Shi S, Shi Q, Ma H, et al. DCN deficiency promotes renal cell carcinoma growth and metastasis through downregulation of P21 and E-cadherin. Tumour Biol (2016) 37:5171-83. doi: 10.1007/s13277-0154160-1

43. Boguslawska J, Rodzik K, Poplawski P, Kedzierska H, Rybicka B, Sokol E, et al. TGF-betal targets a microRNA network that regulates cellular adhesion and migration in renal cancer. Cancer Lett (2018) 412:155-69. doi: 10.1016/ j.canlet.2017.10.019

44. Zhang S, Guo Z, Xu J, Wang J, Zhang J, Cui L, et al. miR-502-mediated histone methyltransferase SET8 expression is associated with clear cell renal cell carcinoma risk. Oncol Lett (2017) 14:7131-38. doi: 10.3892/ ol.2017.7115

45. Liu Q, Guan Y, Li Z, Wang Y, Liu Y, Cui R, et al. miR-504 suppresses mesenchymal phenotype of glioblastoma by directly targeting the FZD7mediated Wnt-beta-catenin pathway. J Exp Clin Cancer Res (2019) 38:358. doi: 10.1186/s13046-019-1370-1

46. Ye MF, Zhang JG, Guo TX, Pan XJ. MiR-504 inhibits cell proliferation and invasion by targeting LOXL2 in non small cell lung cancer. BioMed Pharmacother (2018) 97:1289-95. doi: 10.1016/j.biopha.2017.11.005

47. Ding B, Yao M, Fan W, Lou W. Whole-transcriptome analysis reveals a potential hsa_circ_0001955/hsa_circ_0000977-mediated miRNA-mRNA regulatory sub-network in colorectal cancer. Aging (Albany NY) (2020) 12:5259-79. doi: 10.18632/aging.102945

48. Zhang J, Lou W. A Key mRNA-miRNA-lncRNA Competing Endogenous RNA Triple Sub-network Linked to Diagnosis and Prognosis of Hepatocellular Carcinoma. Front Oncol (2020) 10:340. doi: 10.3389/ fonc. 2020.00340

49. He Y, Jiang Z, Tong F, Li M, Yin X, Hu S, et al. Experimental study of peripheral-blood pro-surfactant protein B for screening non-small cell lung cancer. Acta Cir Bras (2017) 32:568-75. doi: 10.1590/s0102865020170070000008

50. Sin DD, Tammemagi CM, Lam S, Barnett MJ, Duan X, Tam A, et al. Prosurfactant protein B as a biomarker for lung cancer prediction. J Clin Oncol (2013) 31:4536-43. doi: 10.1200/JCO.2013.50.6105

51. Tagne JB, Mohtar OR, Campbell JD, Lakshminarayanan M, Huang J, Hinds AC, et al. Transcription factor and microRNA interactions in lung cells: an inhibitory link between NK2 homeobox 1, miR-200c and the developmental and oncogenic factors Nfib and Myb. Respir Res (2015) 16:22. doi: 10.1186/ s12931-015-0186-6

Conflict of Interest: The authors declare that the research was conducted in the absence of any commercial or financial relationships that could be construed as a potential conflict of interest.

Copyright $\odot 2021$ Yang, Miao, Bai, Tu, Shen, Niu, Xia, Wang and Zhang. This is an open-access article distributed under the terms of the Creative Commons Attribution License (CC BY). The use, distribution or reproduction in other forums is permitted, provided the original author(s) and the copyright owner(s) are credited and that the original publication in this journal is cited, in accordance with accepted academic practice. No use, distribution or reproduction is permitted which does not comply with these terms. 